\title{
Antioxidant Assay Guided Separation of the Methanol Stem Extracts of Binahong (Anredera Cordifolia) Using Cyclic Voltammetry Method
}

\author{
NOVIANY ${ }^{1 *}$, RATU DWI GUSTIA RASYIDI ${ }^{1}$ and ANDI SETIAWAN ${ }^{1}$ \\ 1Department of Chemistry, Faculty of Matematics and Natural Sciences, \\ University of Lampung, 35145, Bandar Lampung, Indonesia. \\ *Corresponding author E-mail: noviany @fmipa.unila.ac.id \\ http://dx.doi.org/10.13005/ojc/330124
}

(Received: December 05, 2016; Accepted: February 02, 2016)

\begin{abstract}
The aim of this study was to identify and characterize groups of antioxidant active naturally from methanol extract of the stem of Anredera cordifolia through antioxidant assay-guided separation. The methanol extract (ME) displayed the highest antioxidant activity, compared to ethyl acetate extract (EE) and hexane extract (HE) using cyclic voltammetry method. Ascorbic acid was used as a positive control in this research. The repeated fractionation of active ME by MPLC and HPLC analyses yielded a constituent with strong antioxidant property. The active constituent (RNV-1) was predicted as a flavonoid type based on the UV spectrum data. The antioxidant activity results exhibited that RNV-1 had a coefficient of antioxidant activity $(\mathrm{K})$ sligtly higher than $\mathrm{K}$ of ascorbic acid with the values of 0.055 and 0.052 respectively. It was found that RNV-1 demonstrated an excellent antioxidant activity in comparison with the standard antioxidant activity through antioxidant assays-guided separation. The results obtained suggested that the stem of $A$. cordifolia can be used potentially as a source of natural antioxidants by contributing beneficial health effects.
\end{abstract}

Keywords: Antioxidant assay-guided separation, Anredera cordifolia, binahong, flavonoid, cyclic voltammetry.

\section{INTRODUCTION}

Interest in plant natural products is certainly undergoing a renaissance at the present time. Many plants have been investigated to identify the phytochemicals and their potential use. Recently, the phenolic compounds particularly flavonoids have received increasing attention among other phytochemicals, due to their wide distribution in the plant kingdom and the various of their biological activities, such as anti-inflammatory, antithrombotic, antiviral, and hepatoprotective as well as their antioxidant activities ${ }^{1,2}$. Furthermore, clinical studies have suggested a positive effect of flavonoids in 
human health and nutrition, such as a decreased risk of heart disease, hormonally dependent cancers, menopausal symptoms, osteoporosis, and cardiovascular disease ${ }^{3}$.

Diets high in flavonoids, fruits, and vegetables are protective against a variety of diseases, in particular cardiovascular disease, and some types of cancer. Antioxidants and dietary fiber are believed to be the principal nutrients responsible for these protective effects. The numerous human health related properties of flavonoids that widely described in epidemiological studies, are mainly based on their antioxidant activities. Antioxidants and dietary fiber are believed to be the principal nutrients responsible for these protective effects ${ }^{4}$.

Nowadays, extensive research on natural antioxidants have been carried out by many researchers ${ }^{5-10}$. The potential antioxidant source can be found also in Anredera cordifolia plant. $A$. cordifolia is one of herbal medicine plant belonging of family Basellaceae. This plant is native to Southern America and is widespread in China, Korea, and Indonesia. The local names of this plant are binahong (Indonesia), Dheng shan chi (China), and madeira vine (England) ${ }^{11}$.
All parts of $A$. cordifolia including leaves, stems, roots and flowers are utilized in folk medicine, but leaves is the most often used. Wahyuni ${ }^{12}$ reported that the leaves extracts of $A$. cordifolia have been shown to produce a variety of compounds such as saponin, alkaloid, phenolic, and flavonoid which exhibited some interesting effects such as antibaterial, anti-inflammation, and antioxidant properties. However, no previous research on the antioxidant activity of the chemical components from the stem part of $A$. cordifolia could be found in the literature. Since this plant has been a valuable traditional remedy for treating infectious diseases therefore, more studies are still needed to further verify the potential of this plant as useful source of antioxidant chemicals.

The antioxidant determination of extracts, fractions, and/or components is currently maintained mainly by DPPH assay. Using an electroanalytical approach for detection of their antioxidant activity is rare $^{13}$. Electrochemical procedures represent advantageous possibility to measure a reduction and oxidation processes in organic compounds that can be helpful in how these compounds are metabolised by living organisms ${ }^{14}$. Recently, Keffous et a/15

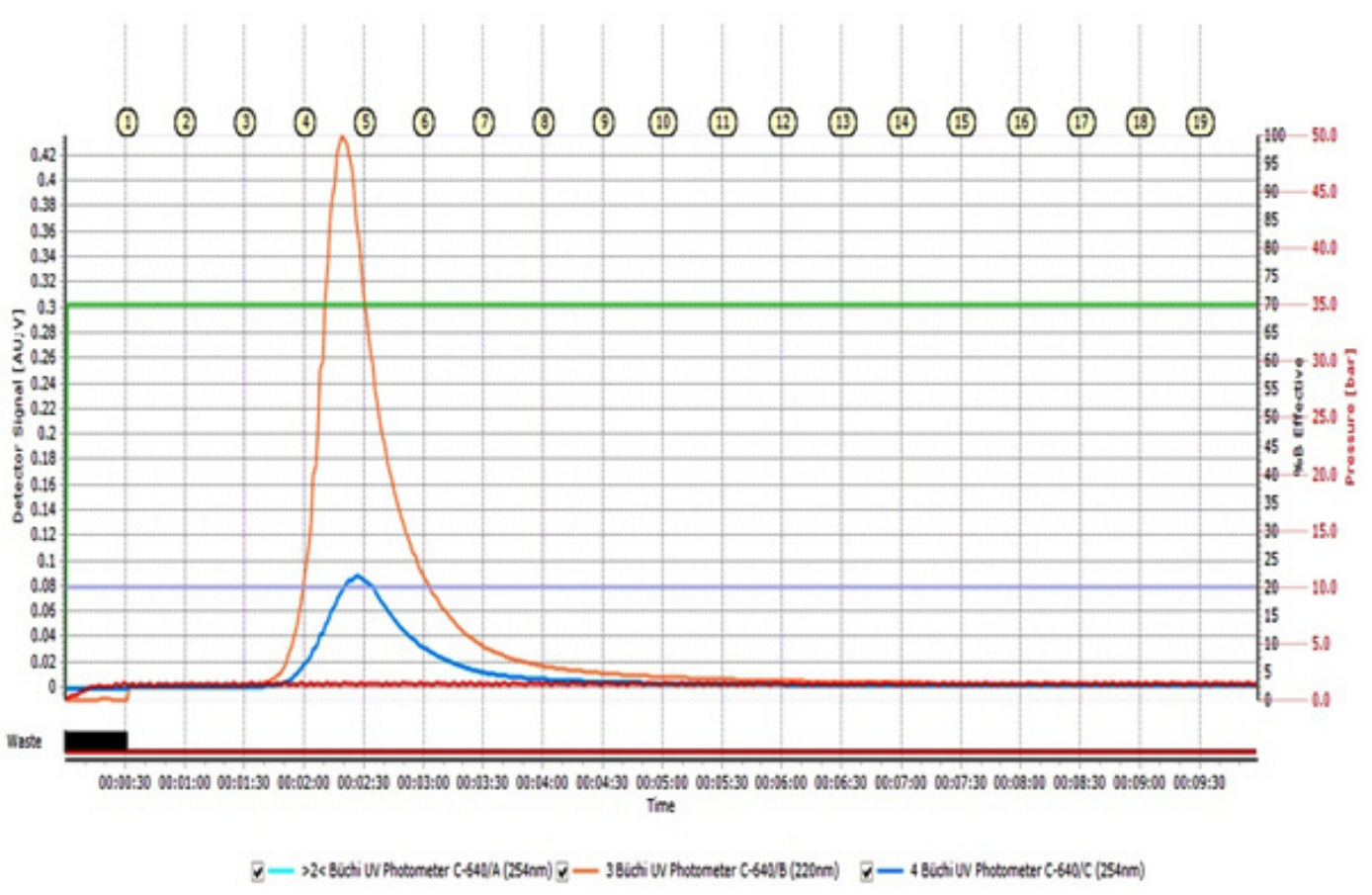

Fig. 1: The MPLC chromatogram of ME of A. cordifolia 
reported the antioxidant activity of Limoniastrum feei aqueous extract by using cyclic voltammetry method. The results showed that the cyclic voltammetry of the extract indicates one oxidation irreversible peak at approximately 300-320 $\mathrm{mV} /(\mathrm{Ag} / \mathrm{AgCl})$. Based on those views, in the present study we sought to evaluate the antioxidant activity of extract and the isolated constituent from the stem of $A$. cordifolia through antioxidant assay-guided separation by using cyclic voltammetry method.

\section{MATERIALS AND METHODS}

\section{Plant material}

The stems of $A$. cordifolia were collected in August 2015 from Bandar Lampung city, Lampung province, Indonesia. The plant specimen was identified at the Herbarium Bogoriense, Research Centre for Biology, Indonesia Institute of Sciences Bogor, Indonesia and a voucher specimen was deposited at the herbarium.

\section{Extraction and isolation}

The dried and powdered stems (500 g) of $A$. cordifolia were extracted with n- hexane, ethyl acetate, and methanol sequentially at room temperature for one week with occasional maceration. Extraction was repeated three times and the fractions were filtered and evaporated using a rotary vacuum evaporator at $40^{\circ} \mathrm{C}$. The weight of each extract were $5.0 \mathrm{~g}, 4.5 \mathrm{~g}$, and $1.3 \mathrm{~g}$ respectively. All the extract obtained were stored at $20^{\circ} \mathrm{C}$ for further analysis. The methanol

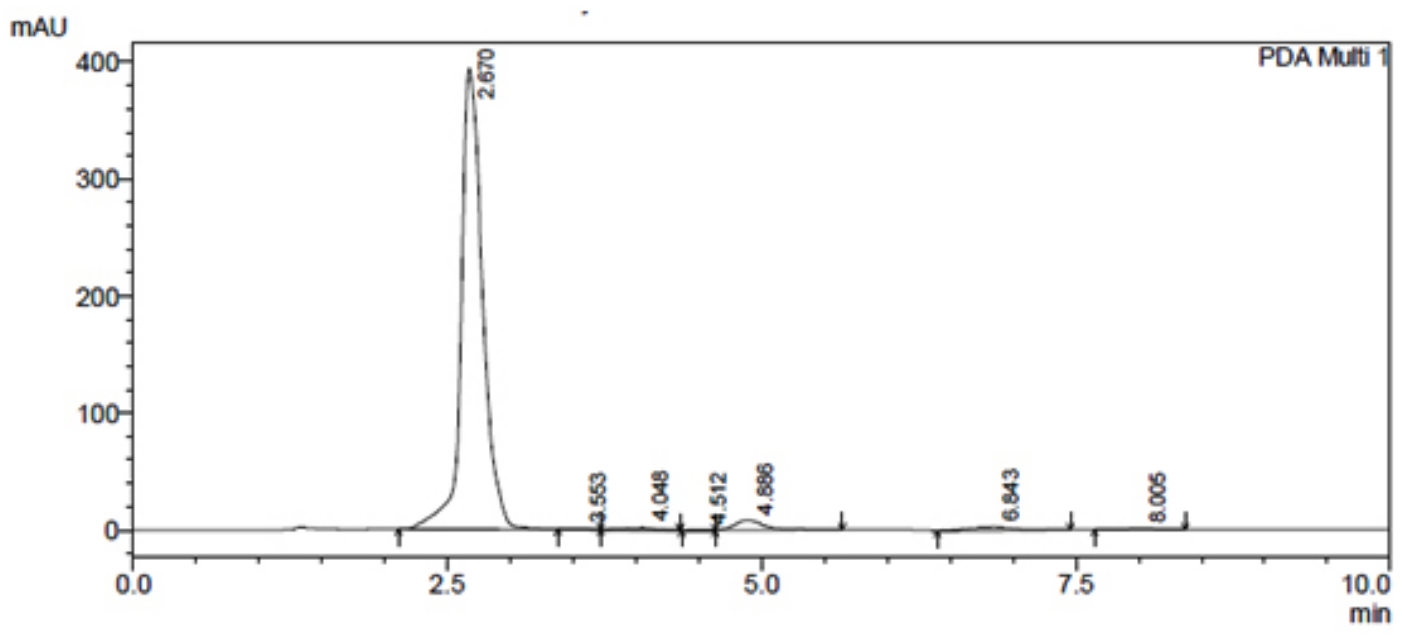

Fig. 2: The HPLC chromatogram of isolated RNV-1

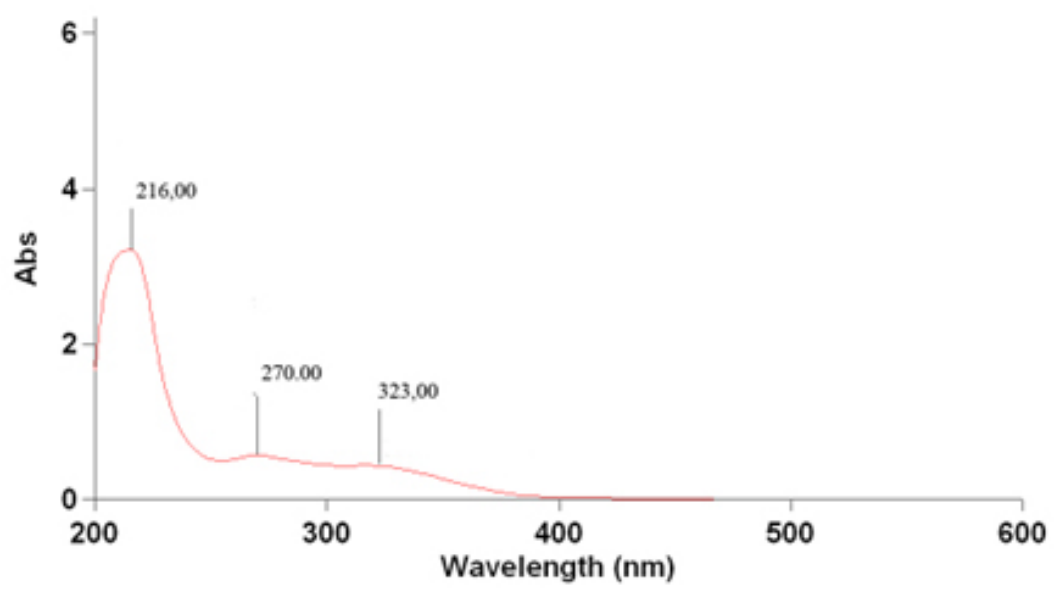

Fig. 3: The UV Spectrum UV of RNV-1 (MeOH) 


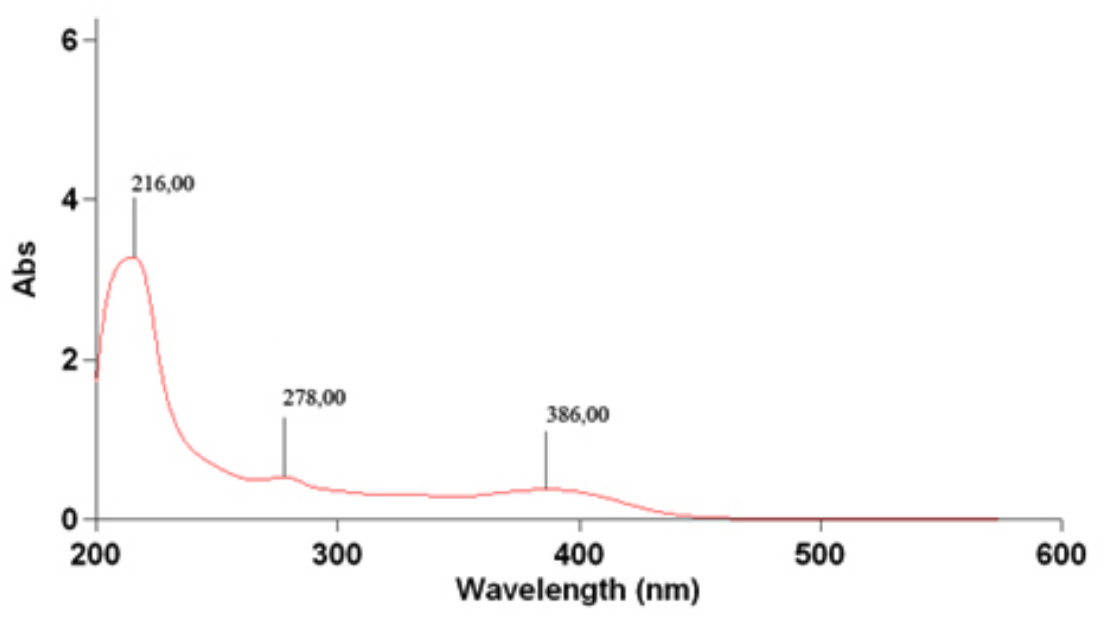

Fig. 4: The UV Spectrum UV of RNV-1 (MeOH+NaOAc/NaOH)

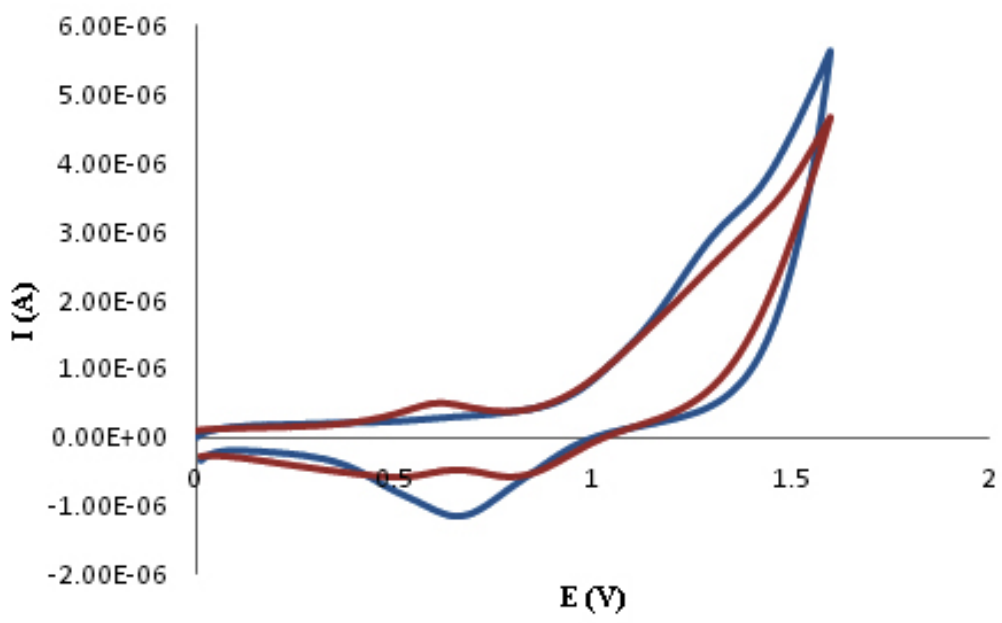

Fig. 5: Cyclic voltammograms of ME recorded at Pt electrode

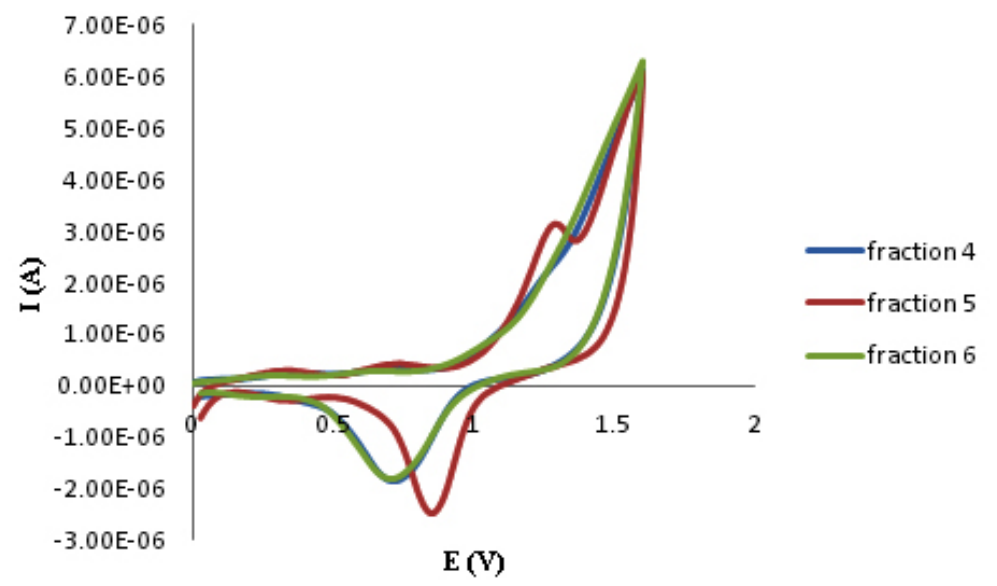

Fig. 6: Cyclic voltammograms of F-4,5,6 of ME recorded at Pt electrode 
extract (ME) was proceeded to fractionation due to its active properties in antioxidant assay using cyclic voltammetry method.

The ME was subjected to Medium Pressure Liquid Chromatography (Buchi/Sepacoterm) with photo diode array (PDA) detector. The analysis were performed with a Cosmosil 75C18-OPN C18 column where methanol-water were used as the mobile phase in a gradient mode (70\%-30\%) at a flow rate of $1.0 \mathrm{~mL} / \mathrm{min}$. The separation processes were repeated for five times and each fraction was analyzed by HPLC (Shimadzu/LC-20A Prominence). The analysis were performed with a Cosmosil Column C18 (150 x 4,6 mm) as follows: methanolxwater $(7: 3)$ was used as the mobile phase; the flow-rate of the mobile phase was $0.1 \mathrm{~mL} / \mathrm{min}$; the effluent was monitored by a wavelength absorbance detector. Characterization of target compound was carried out using Spectroscopy UV-Vis analysis (Agilent UV-Vis Cary 100 Spectrofotometer).

\section{Antioxidant Assay}

All the extracts obtained were evaluated for their antioxidant activity by using cyclic voltammetry method as described by Masek et $\mathrm{al}^{14}$. The most active extract was selected to further fractionate with MPLC analysis. The cyclic voltammetry (CV) method was used, employing an potensiostat-galvanostat analytical unit (eDAQ Pty Ltd) with three-electrode system. Platinum was used as anode and auxiliary electrode. The potential of the tested electrode

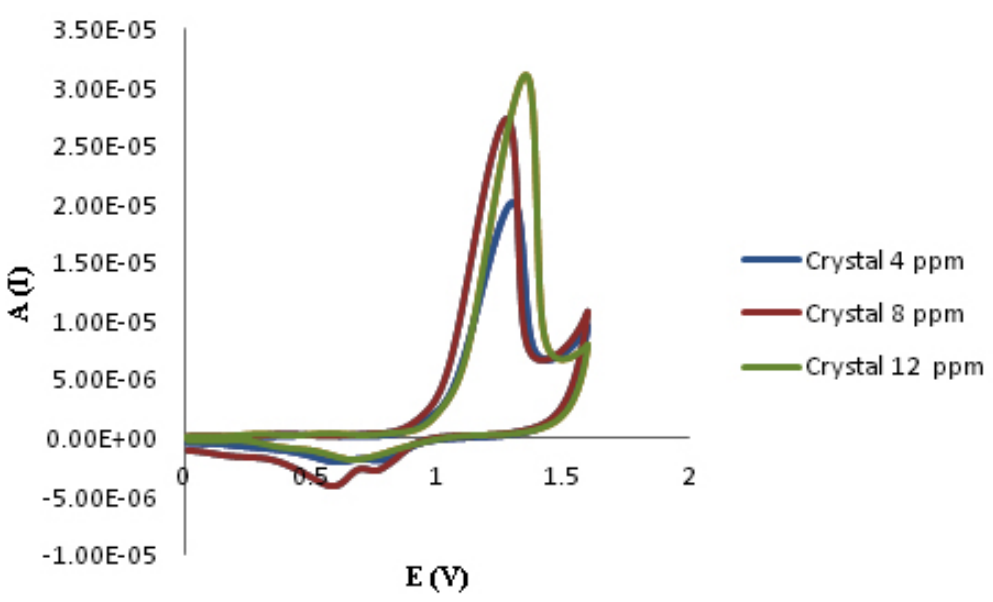

Fig. 7: Cyclic voltammograms of RNV-1 recorded at Pt electrode

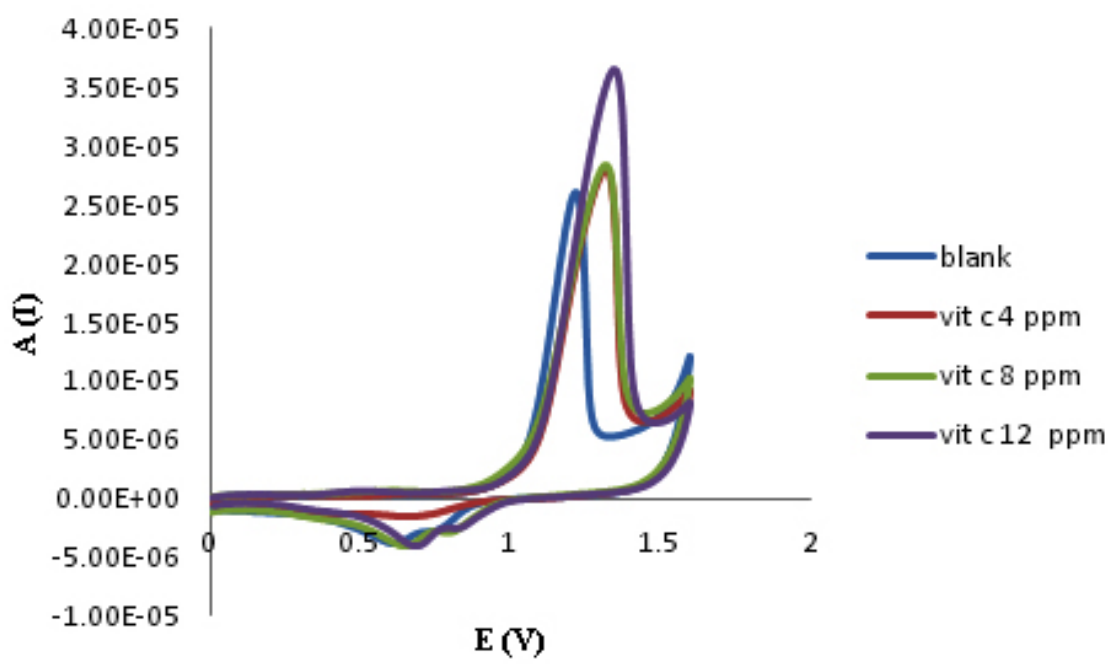

Fig. 8: Cyclic voltammograms of ascorbic acid recorded at Pt electrode 
was measured versus a silver/silver chloride reference electrode. Potassium chlorida from Sigma Aldrich was used a supporting electrolyte and ascorbic acid (Merck) was used as positive control. Prior to measurements, all the solutions were deoxidised with nitrogen. The effect of scan rate on the electrooxidation of tested components in an anhydrous medium was assessed.

\section{RESULTS AND DISCUSSION}

\section{Isolation and Characterization}

All the extracts including $n$-hexane (HE), ethyl acetate (EE), and methanol (ME) were screened for their antioxidant activity using cyclic voltammetry technique. The results demonstrated that the ME has the most active property in antioxidant assay among HE and EE. Therefore, the ME was subjected to fractionation further and purification by MPLC and HPLC analyses. The results of MPLC separation gave 19 fractions. The major component was detected UV detector at 220 and $254 \mathrm{~nm}$ and collected at the retention time 2.5 minutes (Fig.1). Each sub-fractions obtained was tested for its antioxidant activity and the results showed that fraction 4, 5, and 6 displayed the higher antioxidant activity compared with other fractions.

Further purification of fractions 4, 5, and 6 were conducted by crystalization for 1-2 days. Compound RNV-1 (10 mg) was afforded by recrystalization from fraction 5 . The HPLC analysis of isolated RNV-1 (Fig. 2) exposed the presence of major sharp peak with a retention time of 2.67 minutes which indicated that the constituent is quite pure for further analysis by spectroscopy method.

The UV spectrum of RNV-1 revealed over the scan range of 200-400 nm (Fig.3). RNV-1 exhibits a maximum absorbance at $216 \mathrm{~nm}$ with additional absorbances at $260 \mathrm{~nm}$ and $323 \mathrm{~nm}$. The results indicated that RNV-1 has a conjugated system in its structure that could be suggested as phenolic type structure such as flavonoids. Accordingly, the presence of flavonoid compounds was identified in this research based on the shift in absorption maximum caused by addition of sodium acetate/ sodium hidroxide. The absorption characteristics of a flavonoid compound may vary depending on the type of complex it forms with the shift reagents used ${ }^{15,16}$. A bathochromic shift of $8 \mathrm{~nm}$ in band II was observed after addition of sodium hidroxide, implying the presence of 7-OH in flavones or flavonols. Similarly, a red shift of $63 \mathrm{~nm}$ in band I can indicate the flavonoids to be 3-hydroxy flavones or 3,5-dihydroxy flavones ${ }^{16}$. Nevertheless, the completed structure analysis is still on going in this research, to confirm the exact structure of purified compound.

\section{Antioxidant Activity}

In this study, the electrode reactions characterizing electrochemical oxidation of tested components at the platinum electrode were evaluated by cyclic voltammetry. Half-wave potential of a peak in a cyclic voltammogram corresponds to a potential of a peak and is characteristic for each of the subsequent step of the investigated electrode reaction $^{14}$. Cyclic voltammograms recorded in ME solution (red-curve) (Fig. 5) show the small wave appear in the potential range from 0.25 to $0.8 \mathrm{~V}$. This wave is relatively low compare with the peak attributed to the flavonoids oxidation as reported by Masek et $a^{14}$, it can be caused by oxidation of impurities such as water and other organic substances in $\mathrm{ME}^{17}$.

Cyclic voltammograms recorded in some fraction 4 (F-4) (blue-curve), 5 (F-5) (red-curve), and 6 (F-6) (green-curve) of ME (Fig. 6) exhibited that $F-5$ has the most active in antioxidant assay. $A$ peak which characterize at least one electrode step of SF-5 electrooxidation in the potential range from 1.0 to $1.5 \mathrm{~V}$ in voltammogram.

In the case of the isolated compound, RNV-1, the cyclic voltammogram recorded in different concentrations $(4,8$, and $12 \mathrm{ppm})$ showed peaks indicating one electrode of RNV-1 electrooxidation in each concentrations with similarly in the potential range from 1.0 to $1.5 \mathrm{~V}$ (Fig.7).

Cyclic voltammogram recorded in positive control, ascorbic (Fig.8), exhibited that ascorbic acid has less active than with RNV-1 in antioxidant assay. This results also supported by determining a coefficient of antioxidant activity $(\mathrm{K})$ for both isolated compound and ascorbic acid. As expected, RNV-1 have demonstrated excellent antioxidant activity in comparison with the standard antioxidant, ascorbic acid. 


\section{CONCLUSIONS}

The ME exhibited the highest antioxidant property, among the fractions. The antioxidant effects of ME justified the folkloric use of this medicinal plant by the natives in clinical management. Antioxidant assay-guided fractionation and purification of ME resulted in the identification of isolated RNV-1 that was suggested as the flavonoid type compound. The antioxidant activity of RNV-1 was verified the potential of this plant as useful source of antioxidant chemicals in treatment of oxidative damages. The structure elucidation of RNV-1 by using various spectroscopy methods is still in progress.

\section{ACKNOWLEDGMENTS}

The authors are grateful to Directorate of Research and Community Services, Directorate General of Higher Education, The Ministry of Research, Technology and Higher Education, Republic of Indonesia that provide fund for this project to be undertaken through Hibah Pascasarjana (Postgraduated Research Grant) Scheme 2016 with contract number of 963/UN26.21/8/PP/2016, 28 October 2016. We also gratefully acknowledge UPT LTSIT of University Lampung for Technical Assistance and HPLC analysis.

\section{REFERENCES}

1. Marin, F. R.; Frutos, M. J.; Perez-Alvarez, J. A.; Martinez-Sanchez, F.; Del Rio, J. A. Studies in Natural Products Chemistry. 2002, 26, 741778.

2. Shahid, M. A; Shahzad, F.; Sobia, A.; Sahai, T.; Tripathi A.; Singh, H.M.; Khan and Umesh. Anti-Infective Agents in Med. Chem. 2009, 8, 211-225.

3. Du, H; Yubi, H., Yixiong, T. Appl Microbiol Biotechnol. 2010, 86, 1293-1312.

4. Grotewold, E. The Science of Flavonoids, Published by Springer Science Business Media, Inc. 2006, 1-3.

5. Saito, K.; Kohno, M.; Yoshizaki, F.; Niwano, Y. Plant Foods Hum. Nutr. 2008, 63 (2), 65-70.

6. Delgado A. J.; Gamero S. E.; Vald'es S. E.; Gonz'alez-G. D. Food Control. 2012, 24 (1-2), 136-141.

7. Sreelatha, S.; Padma, P. R. Plant Foods Hum. Nutr. 2009, 64 (4), 303-311.

8. Onoja, S. O.; Anaga, A. O. Int. J. Pharm. Phytochem. Res. 2015, 7(2), 340-346.

9. Arullappan, S.; Rajamanickam, P.; Thevar, N.; Narayanasamy, D.; Yee, Y. H.; Kaur, P.; Mahandan, M. Trop. J. Pharma. Res. 2015, 14 (7), 1199-1205.
10. Sudha, A.; Srinivasan, P. BioMed Res. Int. 2014, 1-10.

11. Lestari, D.; Sukandar, E. Y.; Fidrianny, I. Int. J. Pharm. Clin. Res. 2015, 7(6), 435-439.

12. Wahjuni, S.. Ind. J. Biomed. Sci. 2014, 8 (1), 4-7.

13. Hanuštiaka, P.; Mikelova, R.; Potìšila, D.; Hodek, P.; Stiborova, M.; Kizek, R. Biomed. Papers. 2005, 149 (1)

14. Masek, A.; Zaborski, M.; Chrzescijanska, E. Food Chem. 2011, 127, 699-704.

15. Keffous, F.; Belboukhari, N.; Sekkoum, K.; Djeradi, H.; Cheriti, A.; Aboul, E.H.Y. Cogent Chemistry. 2016, 2, 118-141.

16. Schütz, K.; Kammerer, D.R.; Carle, R.; Schieber, A. Rapid Commun. Mass Spectrom. 2005, 19, 179-186.

17. Mabry, T. J.; Markham, K. R.; Thomas, M. B. The Systematic Identification of Flavonoids, Published by Springer-Verlag New York, USA. 1970, 354.

18. Zieja, J.; Gadomska, T. J.; Stojek, Z. Electroanalysis. 2001, 13(8/9), 621-625. 